\title{
Transmission Index Research of Parallel Manipulators Based on Matrix Orthogonal Degree
}

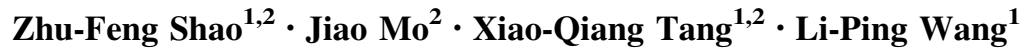

Received: 7 March 2016/Revised: 15 February 2017 / Accepted: 11 October 2017/Published online: 23 October 2017

(c) The Author(s) 2017. This article is an open access publication

\begin{abstract}
Performance index is the standard of performance evaluation, and is the foundation of both performance analysis and optimal design for the parallel manipulator. Seeking the suitable kinematic indices is always an important and challenging issue for the parallel manipulator. So far, there are extensive studies in this field, but few existing indices can meet all the requirements, such as simple, intuitive, and universal. To solve this problem, the matrix orthogonal degree is adopted, and generalized transmission indices that can evaluate motion/force transmissibility of fully parallel manipulators are proposed. Transmission performance analysis of typical branches, end effectors, and parallel manipulators is given to illustrate proposed indices and analysis methodology. Simulation and analysis results reveal that proposed transmission indices possess significant advantages, such as normalized finite (ranging from 0 to 1 ), dimensionally homogeneous, frame-free, intuitive and easy to calculate. Besides, proposed indices well indicate the good transmission region and relativity to the singularity with better resolution than the traditional local conditioning index, and provide a
\end{abstract}

Supported by National Natural Science Foundation of China (Grant Nos. 51575292, 51475252, 91648107), National Key Technology Research and Development Program of China (Grant No. 2105BAF19B00), and National Science and Technology Major Project of China (Grant No. 2016ZX04004004).

Zhu-Feng Shao

shaozf@mail.tsinghua.edu.cn

1 State Key Laboratory of Tribology and Institute of Manufacturing Engineering, Tsinghua University, Beijing 100084, China

2 Beijing Key Lab of Precision/Ultra-precision Manufacturing Equipments and Control, Tsinghua University, Beijing 100084, China novel tool for kinematic analysis and optimal design of fully parallel manipulators.

Keywords Transmission index $\cdot$ Parallel mechanism . Kinematic performance $\cdot$ Matrix orthogonal degree

\section{Introduction}

Parallel manipulator usually consists of the base and the end effector, which are connected together with at least two identical kinematic chains (branches) [1,2]. With the structure feature of closed-loop kinematic chains, the parallel mechanism is recognized as a significant complementary to the traditional serial mechanism [3]. Correspondingly, parallel manipulators possess stable, compact and simple structures with low moving inertia and mass, and show advantages in terms of rigidity, precision, load, and speed, according to Mrthy's theory [4]. Above statement can be verified by the applications as machine tools [5], positioning devices [6], motion simulators [7] and high-speed pick-and-place robots [8,9]. Although application number and field of parallel manipulators increase rapidly, the large-scale commercial application is scarce. As Merlet [10] once pointed out, the performance advantages of parallel manipulators seem to be just potential for now. The industrial performance of the parallel manipulator is one of the most concerned issues for both researchers and engineers. Thus, further study on performance analysis and optimal design should be carried out.

Performance index is the standard to evaluate the manipulator capability and the basis for performance analysis and optimal design. A good index is expected to be finite, independent of the coordinate system, dimensionally homogeneous and with clear physical significance. 
Besides, ease of calculation and intuitive concept will make the index more convenient and likely to be implemented. However, even without considering the mechanism dynamics and elasticity of the parallel manipulator, it is still a challenging issue to seek the suitable kinematic indices [11]. Nature of the mechanism is transmitting force and motion [12], and the parallel mechanism is not an exception. Force/motion transmissibility is the key to the kinematic performance for the parallel mechanism. Intensive efforts have been made to study related indices, which can be classified into three categories.

Index researches with the Jacobian matrix belong to the first category. In kinematics of non-redundant parallel manipulators, force Jacobian matrix is the transpose of kinematic Jacobian matrix, which reflects the truth that force and motion are two equivalent aspects of transmissibility. Based on the condition number of the matrix, Local Conditioning Index (LCI) [13, 14] and Global Conditioning Index (GCI) [15] of the parallel manipulator are developed, which are originally proposed for the serial mechanism [16]. When these indices are applied to the parallel manipulator with combined translational and rotational Degrees of Freedoms (DOFs), dimensional inconsistency appears, which leads to evaluation failure on performance [17, 18]. Then, characteristic length-based method [19] and characteristic point-based method [20] are investigated to formulate the dimensionally homogeneous Jacobian matrix. Recently, MA et al. [21] and Liu et al. [22] found that LCI values could converge when the index is applied to the translational parallel manipulator, which results in poor performance resolution. In addition, the LCI value is frame-related [23, 24].

The second category is referred as angle-based indices, such as pressure angle and transmission angle. Pressure angle is the angle between driving force vector and the velocity vector of force application point. Transmission angle is the complementary angle of the pressure angle, which is defined by the angle between input and output forces of a joint [25]. These indicators are powerful for the planar mechanism [26] with clear significance. Huang et al. [27] evaluated the performance of a two DOFs translational parallel manipulator with the transmission angle. The sine value of the transmission angle is adopted to develop a global force transmission index, and has been applied to performance analysis of the planar $5 \mathrm{R}$ parallel manipulator [28]. Philipp et al. carried out the transmission angle analysis of the planar seven-bar mechanism [29]. Based on the fictitious limb composed of two physical limbs, transmission angles among limbs were proposed, attempting to extend the application of the transmission angle to the spatial parallel mechanism [30]. However, with the increase in limb number as well as the transformation from planar to spatial mechanism, definitions of angle-based indices become difficult and complex with vague physical meanings.

The last category is the transmission indices based on the screw theory $[31,32]$. Considering the virtual coefficient is dimensionless and independent of the coordinate system, the virtual coefficient between input and output wrench screws is defined as the transmission factor, and used to evaluate kinematic performance of the spatial mechanism [33]. Then, the transmission factor is unitized [34], and the generalized transmission wrench screw is proposed [35, 36]. Chen et al. introduced the generalized transmission index and analyzed spatial linkages [37]. Further, input and output transmission indices as well as the total transmission index are proposed [38]. Above indices focus on the analysis of the input power efficiency of the driving joint and the output power efficiency of the branch. The power is calculated through the reciprocal product of twist and wrench screws. Screw theory is a powerful and systematic mathematical tool. However, definition and calculation of these indices are quite complicated, and not intuitive.

There are demands for simple and intuitive force/motion transmission indices for parallel manipulators, considering both planar and spatial mechanism. In this paper, based on the concept of the matrix (or vector group) orthogonal degree, a series of transmission indices are proposed, in terms of the mathematical description, definition and calculation for the fully parallel manipulator, which is considered as the non-redundant parallel manipulator with one actuator in each limb. Then, transmission performance analysis of typical parallel mechanisms is carried out to illustrate proposed indices and the analysis method. The remainder of this paper is organized as follows. In the next section, branch and end-effector transmission indices are introduced, and the local transmission index is defined to evaluate the overall transmissibility of parallel manipulators. Transmission performance analysis of typical branches and end effectors for fully parallel manipulators are illustrated in sections 3 and 4 . In section 5, transmissibility of two typical parallel manipulators is discussed. Conclusions of this paper are given in section 6 .

\section{Mathematical Foundation and Index Definition}

In this paper, transmission indices are deduced on the basis of orthogonal degree of matrix, which is defined with matrix volume [39]. First of all, matrix volume can be described as follows: assuming $\boldsymbol{X}_{m \times n}=\left[\begin{array}{llll}\boldsymbol{x}_{1} & \boldsymbol{x}_{2} & \ldots & \boldsymbol{x}_{n}\end{array}\right]$ is a real matrix (column vector group), composed of $n$ real column vectors $\boldsymbol{x}_{i}$. Then, the volume of the real matrix can be deduced as 
$\operatorname{vol}_{n}(\boldsymbol{X})=\sqrt{\operatorname{det}\left(\boldsymbol{X}^{\mathrm{T}} \boldsymbol{X}\right)}$.

Further, the matrix orthogonal degree can be described as following: if $\min \left\|\boldsymbol{x}_{i}\right\|=0(i=1,2, \ldots, n)$, the matrix orthogonal degree $\operatorname{ort}(\boldsymbol{X})$ equals 0 . Otherwise,

$\operatorname{ort}(\boldsymbol{X})=\operatorname{vol}_{n}(\boldsymbol{X}) / \prod_{i=1}^{n}\left\|\boldsymbol{x}_{i}\right\|=\sqrt{\operatorname{det}\left(\boldsymbol{X}^{\mathrm{T}} \boldsymbol{X}\right)} / \prod_{i=1}^{n}\left\|\boldsymbol{x}_{i}\right\|$.

Above definition is based on the column vector, and can be considered as the volume of matrix composed of unit column vectors. On the basis of above definition, it is easy to get the property that $0 \leq \operatorname{ort}(\boldsymbol{X}) \leq 1$. When column vectors are orthogonal to each other, the maximum value $1 \mathrm{can}$ be obtained. On the contrary, when multicollinearity of column vectors appears, the minimum value 0 can be deduced. Extreme values of the matrix orthogonal degree indicate two important geometric properties, multicollinearity and orthogonality of multiple vectors. Obviously, matrix orthogonal degree is an efficient mathematical tool to study force/motion vectors of spatial parallel mechanisms.

The force (or motion) transmission in mechanism could be understood as from the base (fixed on the ground) to the terminal. According to structural features of the parallel mechanism, identical branches connect the end effector with the base. Transmissibility of the parallel manipulator can be subdivided into two aspects, such as branch transmissibility and end-effector transmissibility. The former describes the manipulability of the limb, and the later indicates the complementarity of limbs' contributions on the end effector.

Firstly, let us focus on the branch transmissibility. Each branch (or limb) of the parallel manipulator is a typical serial mechanism, and force is transmitted from the base to the end effector through the limb. Limb transmissibility depends on transmission performances of the mounted joints. Index of the joint transmissibility can be defined by the relation between the afferent force applying on the joint by the previous component and the efferent force that exerted on the next component through the joint. Specifically, Joint Transmission Index (JTI) of the $i$ th joint located in the $j$ th branch of the parallel manipulator can be written as

$\Gamma_{\mathrm{JTI} j i}=\sqrt{1-\operatorname{ort}\left(\boldsymbol{Y}_{j i}\right)^{2}}$,

where $\boldsymbol{Y}_{j i}=\left[\begin{array}{ll}F_{j i \mathrm{~A}} & F_{j i \mathrm{E}}\end{array}\right] . \boldsymbol{f}_{j i \mathrm{~A}}$ and $\boldsymbol{f}_{j \mathrm{iE}}$ are the afferent and efferent force vectors, respectively. From the above definition, it can be found that when these force vectors are collinear, the force transmissibility is the best, and $\Gamma_{\mathrm{JTI}}=1$. On the contrary, when force vectors are mutually perpendicular, the force transmissibility is the worst, and $\Gamma_{\mathrm{JTI}}=0$.
Numerically, the JTI value equals the cosine value of the pressure angle, and indicates the ratio of the efferent force to the afferent force. For different limbs, JTI analysis is diverse. The JTI descriptions of typical branches of parallel manipulators will be introduced in Section 3.

The branch of the parallel manipulator is formed by a series of joints. On the basis of the JTI, the Branch Transmission Index (BTI) can be defined as the product of JTI values of all joints in the branch, and can be written as $\Gamma_{\mathrm{BTI} j}=\Gamma_{\mathrm{JTI} j 1} \times \Gamma_{\mathrm{JTI} j 2} \times \cdots \times \Gamma_{\mathrm{JTI} j k}$.

Eq. (4) reflects the overall transmission performance of the limb from the base to the end-effector joints. When the BTI value of a branch equals 0 , the end effector of the parallel manipulator will lose the support of the branch. In this case, the manipulator is at its singular configuration, and this kind of singularity is called the reverse singularity.

The other aspect of transmission analysis for the parallel manipulator is the end-effector transmissibility, which reflects the integrated efficacy of all branches. The Endeffector Transmission Index (ETI) reflects the ability of branch force vectors to span the whole required output force space, and is defined with the limb force vectors exerted on the end effector and the end-effector geometric characteristics. The ETI can be calculated as

$\Gamma_{\mathrm{ETI}}=\operatorname{ort}(\boldsymbol{E F})$,

where $\boldsymbol{F}=\left[\begin{array}{llll}\tau_{1} & \tau_{2} & \ldots & \tau_{n}\end{array}\right]$ is the branch force matrix. The limb force vector of the $j$ th branch is $\tau_{j}=\left[\boldsymbol{f}_{j} ; \boldsymbol{c}_{j} \times \boldsymbol{f}_{j}\right]$, composed of the unit force vector $f_{j}$ and unit torque vector $\boldsymbol{c}_{j} \times \boldsymbol{f}_{j}, \boldsymbol{f}_{j}$ is the unit force vector exerted on the end effector by the $j$ th limb, while $\boldsymbol{c}_{j}$ is the unit radial vector pointing from the geometric center of the end effector to the rotational center of the end-effector joint of the $j$ th limb. Rigid body in space possesses six DOFs, but some DOFs of the end effector are constrained to obtain the required DOFs. The matrix $\boldsymbol{E}$ is a diagonal matrix, used to describe the required DOFs of the parallel manipulator. When a degree of freedom of the end effector is required and movable, the corresponding diagonal element of matrix $\boldsymbol{E}$ is 1. On the contrary, if some DOFs are constrained, the corresponding diagonal elements of matrix $\boldsymbol{E}$ are 0. Take the 3-RRR parallel manipulator for example. The matrix $\boldsymbol{E}=\operatorname{diag}(1,1,0,0,0,1)$, which indicates the end effector of this manipulator possesses two translational DOFs along $X$ and $Y$ axes and a rotational degree of freedom along $C$ axis (represents the rotational movement around the $Z$ axis). The dimension of the matrix $\boldsymbol{E F}$ always equals the number of required end-effector DOFs, and the ETI is applicable to all non-redundant parallel manipulator. If the end effector of the parallel manipulator is a point-type, for example 5R parallel manipulator, $\boldsymbol{c}_{j}$ becomes zero. It is 
worth noting that when the value of ETI is 0 , the parallel manipulator will lose the force output in a certain direction. In this case, the manipulator is at its forward singularity.

As mentioned above, force transmission in the parallel manipulator can be considered as from the base to the end effector. Thus, the total transmissibility of the parallel manipulator equals the product of BTI and ETI values. However, BTI values and branch transmission performances are different among various branches under the arbitrary pose. Considering that the main work in the design and analysis stage is to avoid configuration and pose with poor transmission performance, the minimal BTI value is adopted, and the Orthogonal degree based Local Transmission Index (OLTI) of the parallel manipulator can be defined as

$\Gamma_{\mathrm{OLTI}}=\min \left(\Gamma_{\mathrm{BTI} j}\right) \cdot \Gamma_{\mathrm{ETI}}(j=1,2, \ldots, n)$.

When the value of OLTI is 0 , the parallel mechanism is in a singularity locus. Conversely, when the value of OLTI is 1 , the transmissibility of the mechanism is the best. As illustrated in the above definitions, the proposed transmission indices are not related to the established coordinate system, and are frame-free. The value range is finite and normalized $[0,1]$. Besides, index significances are clear, while calculation is simple and concise.

\section{Transmissibility of Typical Branches}

In this section, based on the proposed BTI, transmission performances of typical branches for fully parallel manipulators are analyzed. Gravity, friction and inertial forces are not taken into account in this paper.

\subsection{Planar RRR and Spatial RSS Branches}

As shown in Figure 1, planar RRR branch ( $\mathrm{R}$ stands for revolute joint, and the underline indicates the actuated joint) is composed of three revolute joints $(1,2$, and 3$)$ as well as two links (I and II). $l_{j 1}$ and $l_{j 2}$ are unit link vectors. Link I is the swing bar, and link II is the two-force bar. Forces are

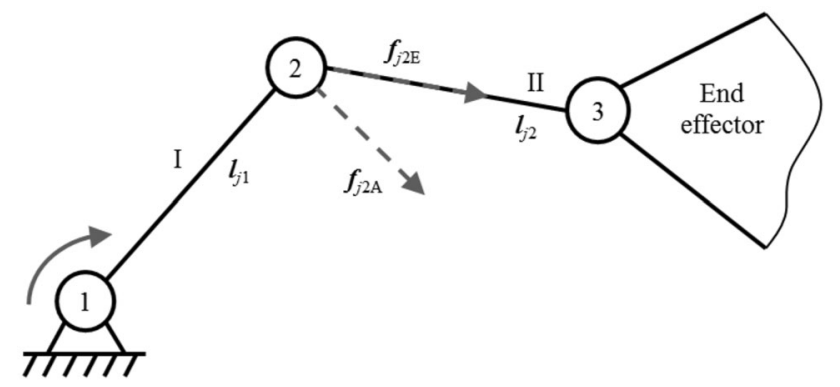

Figure 1 Planar RRR branch indicated with dotted-line arrows, while actuated joint is labeled with the solid-line arrow. The actuated revolute joint is attached to the base, and the JTI value of the joint is 1 . For the end-effector joint, the JTI value of joint 3 is also 1 . The transmission performance of the branch is completely determined by joint 2 . As indicated in the figure, the afferent force of joint 2 is $f_{j 2 \mathrm{~A}}$, perpendicular to the link I. The efferent force of joint 2 is $f_{j 2 \mathrm{E}}$, along the link II. Therefore, the BTI value of the $j$ th branch can be determined as

$\Gamma_{\mathrm{BTI} j}=\Gamma_{\mathrm{JTI} 2}=\sqrt{1-\operatorname{ort}\left(\boldsymbol{Y}_{j 2}\right)^{2}}$,

and $\boldsymbol{Y}_{j 2}=\left[\begin{array}{ll}\boldsymbol{f}_{j 2 \mathrm{~A}} & \boldsymbol{f}_{j 2 \mathrm{E}}\end{array}\right]$. According to the geometric relation, the BTI value of the RRR branch can be directly calculated with structural vectors as $\Gamma_{\mathrm{BTI} j}=\Gamma_{\mathrm{JTI} 2}=$ ort $\left(\boldsymbol{L}_{j}\right)$, and $\boldsymbol{L}_{j}=\left[\begin{array}{ll}\boldsymbol{l}_{j 1} & \boldsymbol{l}_{j 2}\end{array}\right]$.

This conclusion is also applicable to the spatial RSS branch (S represents the spherical joint), as shown in Figure 2. The actuated joint of the RSS branch is also the revolute joint attached to the base. The link II is the twoforce bar, and values of JTI for actuated and end-effector joints are both 1 . The transmission performance of the branch is determined by the middle joint. Then, the BTI value of the RSS branch can be described as

$\Gamma_{\mathrm{BTI} j}=\Gamma_{\mathrm{JTI} j 2}=\sqrt{1-\operatorname{ort}\left(\boldsymbol{Y}_{j 2}\right)^{2}}=\operatorname{ort}\left(\boldsymbol{L}_{j}\right)$.

For the RSS branch, the link vectors become three-dimensional. Generally, above analysis and calculation process is based on force vectors, and has nothing to do with the definition of the coordinate system. Above conclusion fits for a class of branches, composed of actuated pendulum and passive two-force bar, such as RUS, RUU and RRS branches (U stands for the universal joint).

\subsection{PUS and UPS Branches}

Besides revolute joint, prismatic joint is also widely adopted as actuated joint, and usually placed on the base or in the middle of the branch. The typical structures are PUS and UPS branches (P represents the prismatic joint). As shown in Figure 3, the universal joint of the PUS branch is in the middle, and the spherical joint is attached to the end

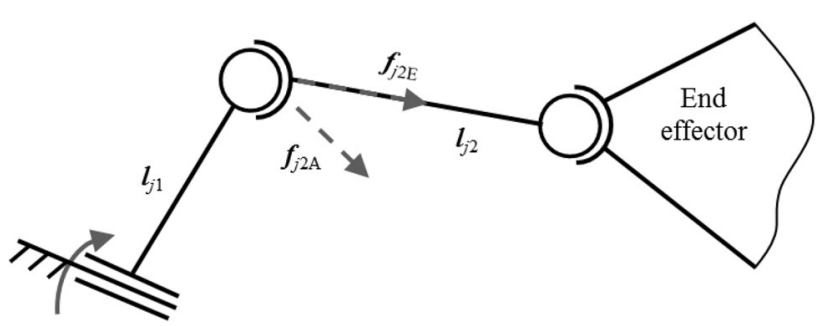

Figure 2 Spatial RSS branch 
effector. The JTI value of the actuated joint is 1 . Link II is a two-force bar, the force acting on the end effector is along link II, and the JTI value of spherical joint (end-effector joint) is 1 . The BTI value of the limb equals the JTI value of the middle universal joint. As shown in Figure 3, the afferent force $f_{j 2 \mathrm{~A}}$ and the efferent force $\boldsymbol{f}_{j 2 \mathrm{E}}$ of the universal joint can be determined, and the BTI value of the PUS branch is deduced as

$\Gamma_{\mathrm{BTIj}}=\Gamma_{\mathrm{JTT} j 2}=\sqrt{1-\operatorname{ort}\left(\boldsymbol{Y}_{j 2}\right)^{2}}=\sqrt{1-\operatorname{ort}\left(\boldsymbol{L}_{j}\right)^{2}}$,

where $\quad \boldsymbol{Y}_{j 2}=\left[\begin{array}{ll}\boldsymbol{f}_{j 2 \mathrm{~A}} & \boldsymbol{f}_{j 2 \mathrm{E}}\end{array}\right]$, and $\boldsymbol{L}_{j 2}=\left[\begin{array}{ll}\boldsymbol{l}_{j 1} & \boldsymbol{l}_{j 2}\end{array}\right]$. This equation is applicable to the branch that consists of actuated prismatic joint attached to the base and passive twoforce bar, such as PSS, $\underline{P R S}$ and PRU branches.

As shown in Figure 4, the actuated prismatic joint of the UPS branch is installed in the middle of the branch. The entire branch becomes the two-force bar. It can be deduced that JTI values of two passive joints are both 1 , and the BTI value of all branches is 1 . This result can be applied to other two-force branches, driven by middle prismatic joint, such as SPS, RPS, and RU branches.

In this section, the transmission performance analysis is carried out on typical branches. Considering the inertia property and power consumption, the actuated joint of the parallel manipulator is usually attached to the base or located in the middle of the branch, then JTI values of the base and actuated joints usually equal 1 . There are many two-force bars in the parallel manipulator, which facilitate and simplify the force analysis and the BTI value calculation greatly.

\section{Transmissibility of End Effectors}

For the parallel manipulator, the base and the end effector are connected with multiple identical branches simultaneously. The output force of the end effector is the common

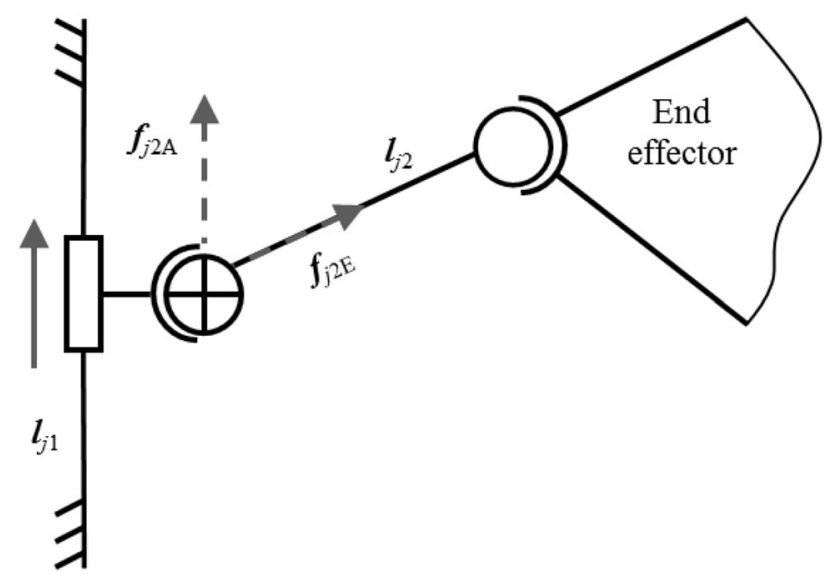

Figure 3 Spatial PUS branch

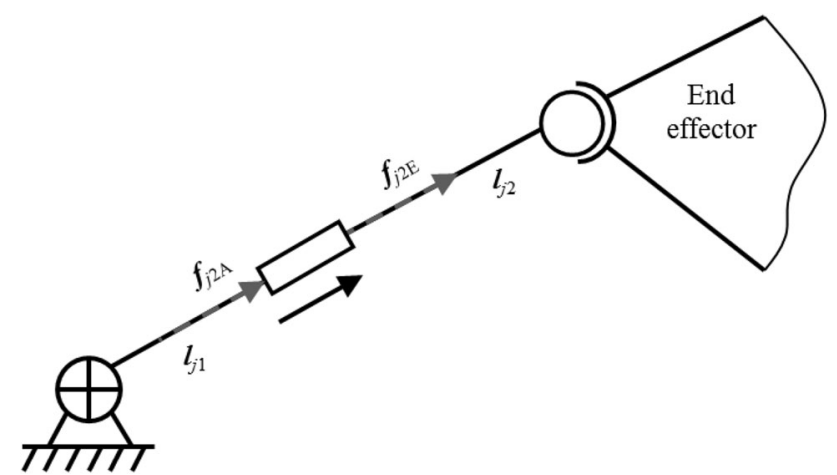

Figure 4 Spatial UPS branch

effort of all branches. The typical planar and spatial parallel mechanisms, such as 5R and Stewart parallel manipulators, are analyzed in this section to reveal the endeffector transmissibility.

The structure of $5 R$ mechanism is shown in Figure 5. Point $\mathrm{P}$ is the end effector, with two translational DOFs. Both branches are of the RRR configuration. According to the definition of ETI proposed in Section 2, the ETI expression of the $5 \mathrm{R}$ mechanism can be written as

$\Gamma_{\mathrm{ETI}}=\operatorname{ort}(\boldsymbol{E F})=\operatorname{ort}\left(\left[\begin{array}{ll}\boldsymbol{f}_{1} & \boldsymbol{f}_{2}\end{array}\right]\right)$,

where $f_{1}$ and $f_{2}$ are unit force vectors exerted on the end effector by two branches, along $l_{12}$ and $l_{22}$ vectors respectively, which are unit structural vectors of passive links. According to the geometric relation, the transmission index can also be written as $\Gamma_{\mathrm{ETI}}=\operatorname{ort}(\boldsymbol{L})$ and $\boldsymbol{L}=\left[\begin{array}{ll}\boldsymbol{l}_{12} & \boldsymbol{l}_{22}\end{array}\right]$. For the 5R parallel manipulator, the ETI value equals the sine value of the angle between $f_{1}$ and $f_{2}$ vectors $\left(\boldsymbol{l}_{12}\right.$ and $\boldsymbol{l}_{22}$ vectors). Obviously, when the angle

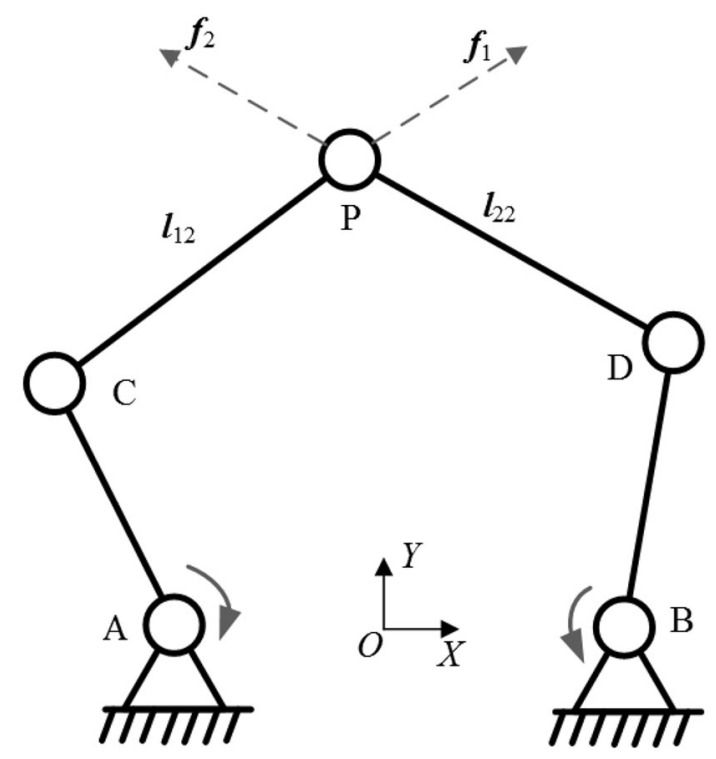

Figure 5 Planar 5R mechanism 
becomes $0^{\circ}$ or $180^{\circ}$, limb force vectors are collinear, and the end effector loses the force output capacity along the direction perpendicular to limb force vectors. Accordingly, a singularity occurs, and the ETI value is 0 . If the angle is $90^{\circ}$, the transmissibility of the end effector is the best, and the ETI value equals 1 .

As shown in Figure 6, the Stewart parallel manipulator consists of six extensible UPS branches, which is a typical 6 DOFs parallel manipulator with combined translational and rotational DOFs. According to the branch analysis, the unit force $f_{j}$ acting on the end effector by the $j$ th limb is along the limb, through rotational center of the spherical joint. As illustrated in Figure 6, the geometric center of the end effector is point $o$. Thus, direction of the unit radial vector $\boldsymbol{c}_{\mathrm{j}}$ is from point $o$ to the rotational center of the $j$ th spherical joint. Considering the geometric structure, the unit branch vector $\boldsymbol{l}_{j 2}$ can be used instead of the limb force vector $\boldsymbol{f}_{j}$. The matrix $\boldsymbol{E}$ becomes the unit diagonal matrix, and all diagonal elements are 1 . Then, we can deduce the $\Gamma_{\text {ETI }}$ expression of the Stewart manipulator as

$\Gamma_{\text {ETI }}=\operatorname{ort}(\boldsymbol{E F})=\operatorname{ort}\left(\left[\begin{array}{rll}\boldsymbol{l}_{12} & \cdots & \boldsymbol{l}_{62} \\ \boldsymbol{c}_{1} \times \boldsymbol{l}_{12} & \cdots & \boldsymbol{c}_{6} \times \boldsymbol{l}_{62}\end{array}\right]\right)$.

And, singularity of the Stewart parallel manipulator will be discussed in the next section.

\section{Examples}

In order to illustrate the analysis method and the proposed indices in detail, the transmission performances of two typical parallel manipulators are analyzed with proposed indices in this section, and comparative discussion with the traditional LCI is developed.

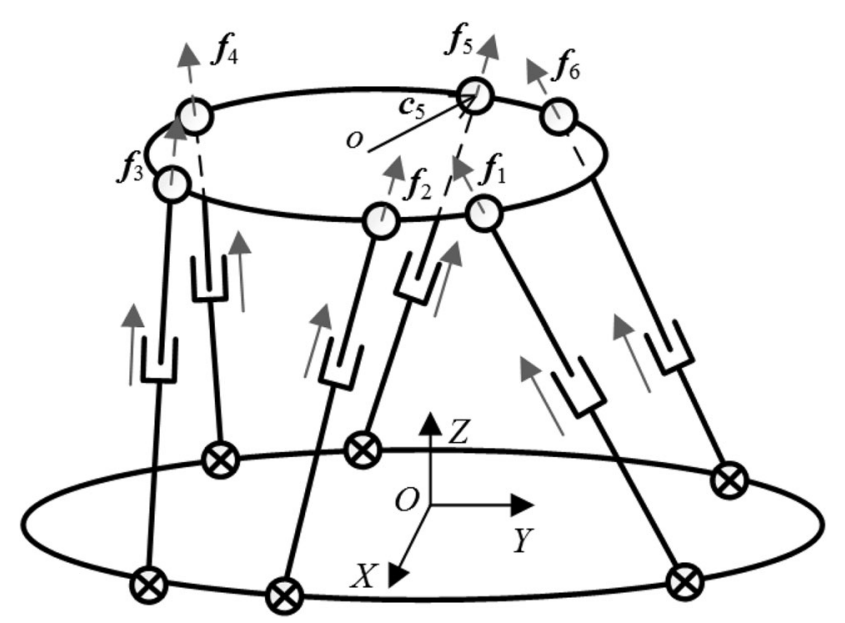

Figure 6 Spatial Stewart parallel manipulator
Firstly, the 5R parallel manipulator is analyzed, and adopted kinematic parameters are listed in Table 1. The global coordinate system is established at the midpoint between two actuated joints, as shown in Figure 5. With Eqs. (6), (7) and (10), value distributions of the proposed indices, such as BTI, ETI and OLTI, can be determined, as shown in Figures 7, 8, and 9 respectively. The value distribution of traditional LCI is exhibited in Figure 10.

As shown in Figure 8, in the reachable workspace (illustrated with red curve), ETI values are relatively large, which indicates that the transmissibility of the end effector for the $5 \mathrm{R}$ mechanism is quite good. With comparative analysis of Figures 7, 8, and 9, we can find that the overall transmission performance of the $5 \mathrm{R}$ mechanism is mainly determined by the branch transmissibility. On the boundary of the reachable workspace, reverse singularity occurrence, introduced by the branch transmission performance, as shown in Figure 7. With the proposed indices, it is convenient to determine the singularity type and analyze the cause.

As shown in Figures 9 and 10, overall trends of LCI and OLTI atlases for the 5R parallel manipulator are similar. Large index values which mean good transmission performance appear in the center of the reachable workspace. With the end effector moves away from the center region, the transmission performance becomes worse. However, there are some significant differences between LCI and

Table 1 Kinematic parameters of the 5R parallel manipulator

\begin{tabular}{lc}
\hline Parameter & Value \\
\hline Horizontal distance of actuated joints $D / \mathrm{m}$ & 1.0 \\
Length of the actuated pendulum $L_{1} / \mathrm{m}$ & 0.8 \\
Length of the passive link $L_{2} / \mathrm{m}$ & 1.6 \\
\hline
\end{tabular}

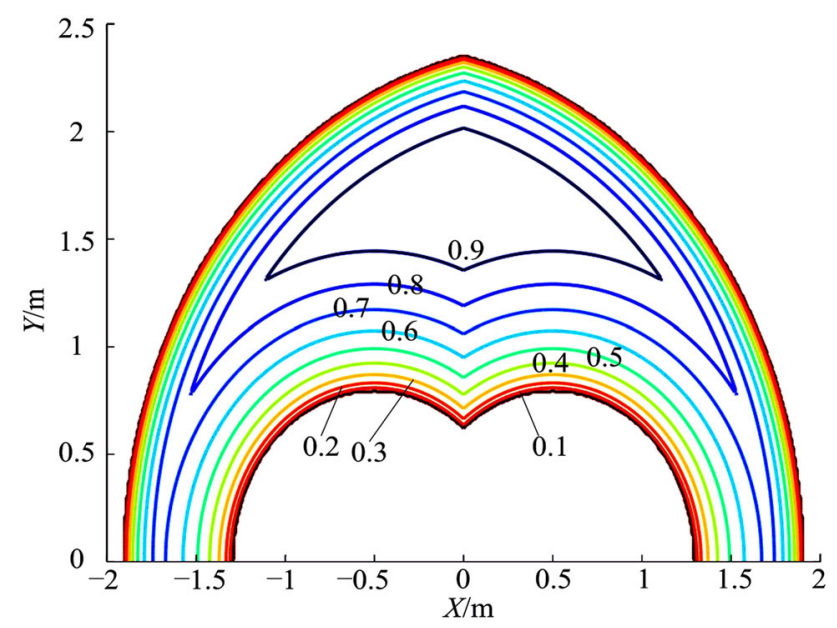

Figure 7 BTI atlas 


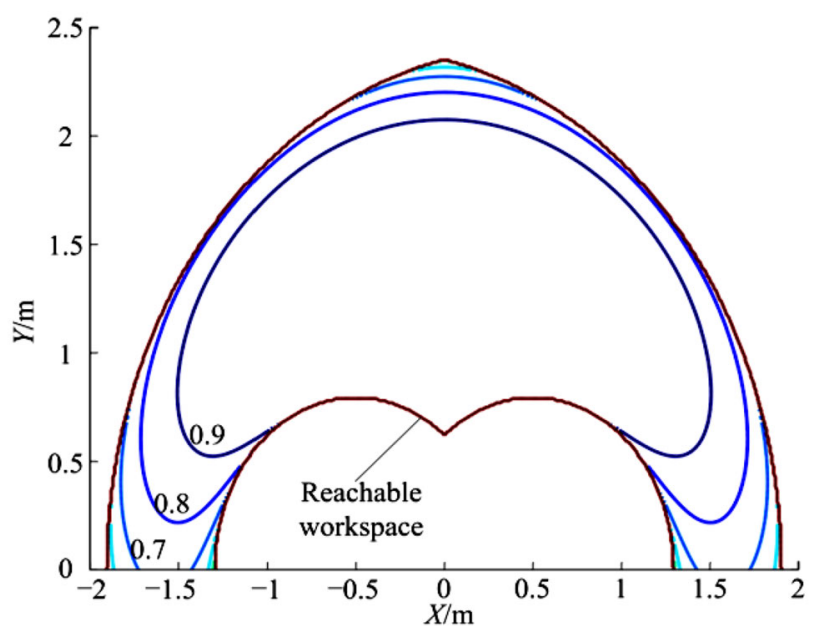

Figure 8 Atlas of $\min \left(\Gamma_{\mathrm{ETI} 1}, \Gamma_{\mathrm{ETI} 2}\right)$

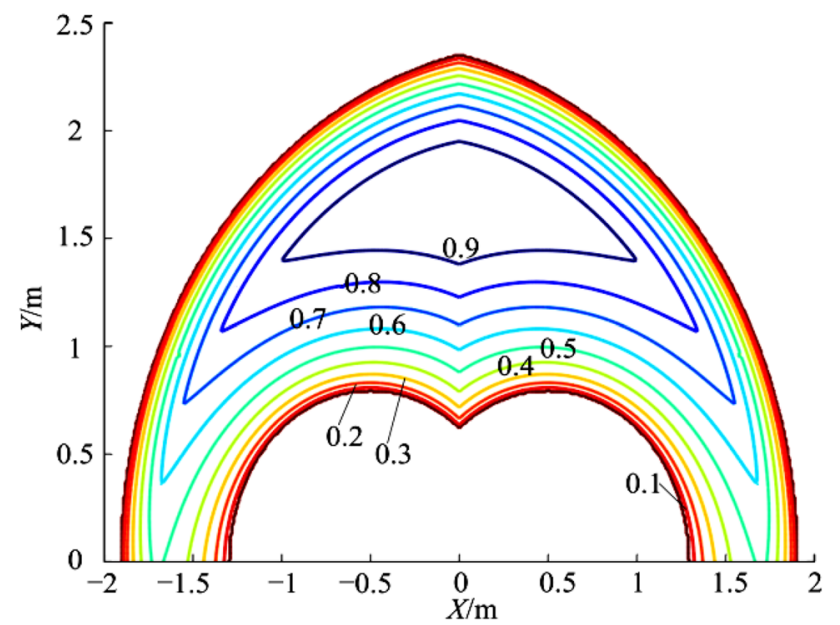

Figure 9 OLTI atlas

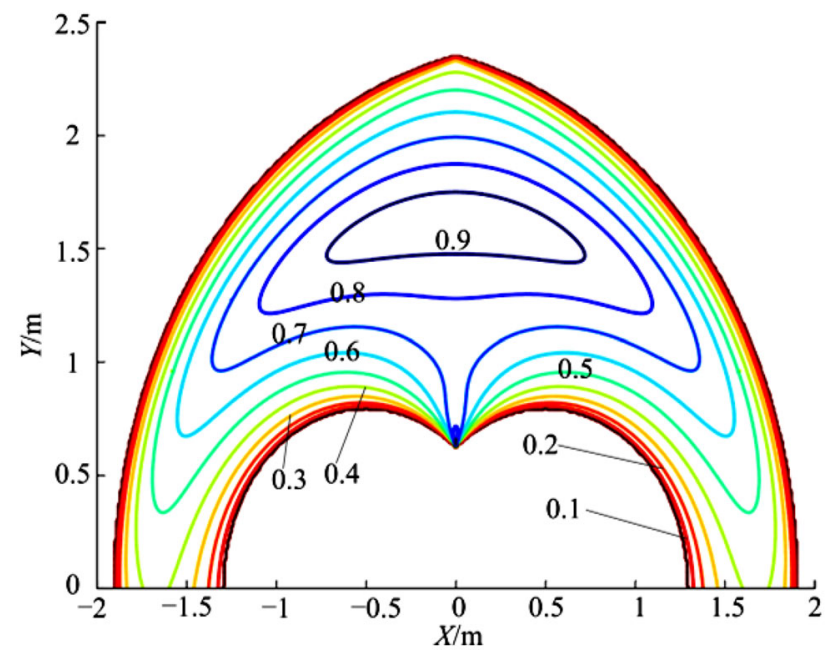

Figure 10 LCI atlas
OLTI atlases. In the LCI atlas, contour curves with different LCI values converge, which reduces the performance resolution capacity and fails to reflect the distance off singularity. In contrast, contour curves with different OLTI values never intersect, and the offset of each curve is quite clear. The proposed index is relative to the singularity.

In addition, the transmission performance of the Stewart parallel manipulator is evaluated, and adopted kinematic parameters are shown in Table 2. Joint distribution angles $\alpha$ and $\beta$ of the Stewart manipulator are used to describe locations of spherical and universal joints in the end effector and the base respectively, as shown in Figure 11. The global coordinate system is established at the geometrical center of the base, as illustrated in Figures 6 and 11.

The distributions of LCI and OLTI for the Stewart parallel manipulator are given in Figures 12 and 13, when the end effector is located in the horizontal plane of $Z=0.45 \mathrm{~m}$ without any rotation. Since the BTI value of the Stewart manipulator is the constant 1, its OLTI atlas is the same with its ETI atlas. In the calculation of LCI value, considering the dimensional homogeneity, the end-effector

Table 2 Kinematic parameters of the Stewart parallel manipulator

\begin{tabular}{ll}
\hline Parameter & Value \\
\hline Radius of the base $R / \mathrm{m}$ & 0.45 \\
Radius of the end effector $r / \mathrm{m}$ & 0.225 \\
Joint distribution angle of the base $\alpha /\left(^{\circ}\right)$ & 10 \\
Joint distribution angle of the end effector $\beta /\left(^{\circ}\right)$ & 25 \\
\hline
\end{tabular}

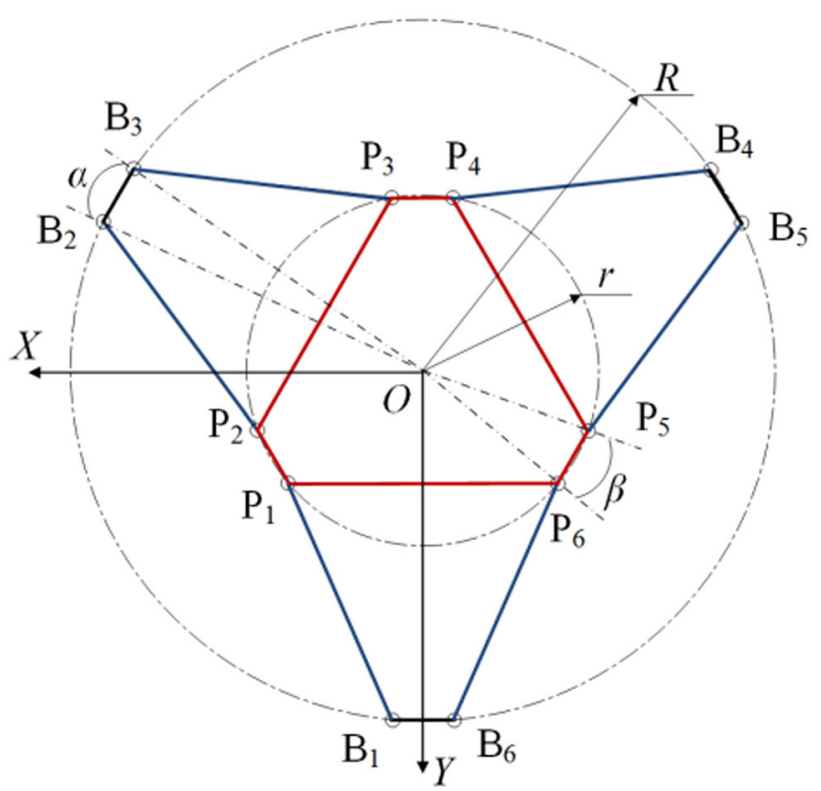

Figure 11 Top view of the Stewart parallel manipulator 


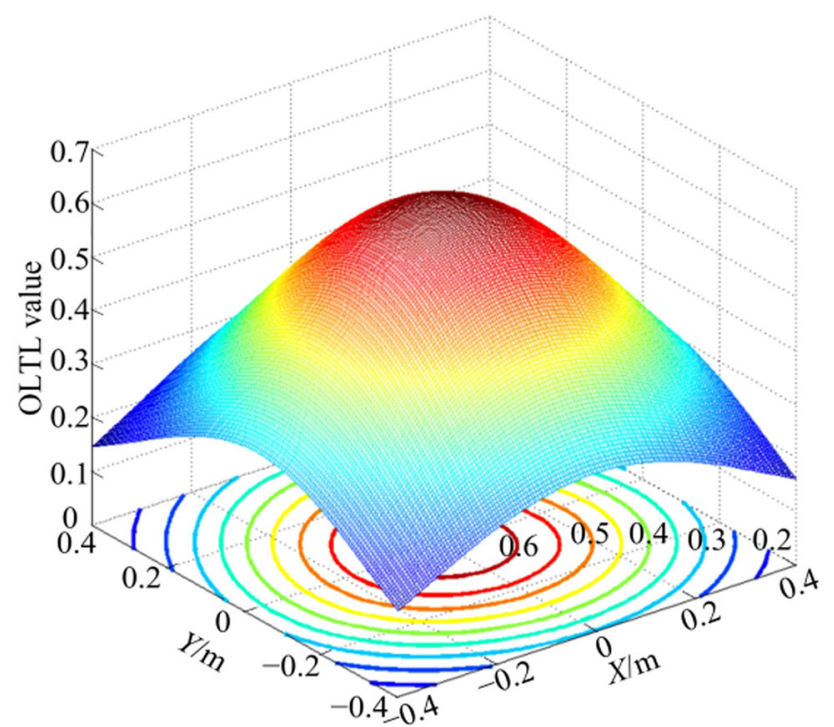

Figure 12 OLTI atlas of the Stewart parallel manipulator

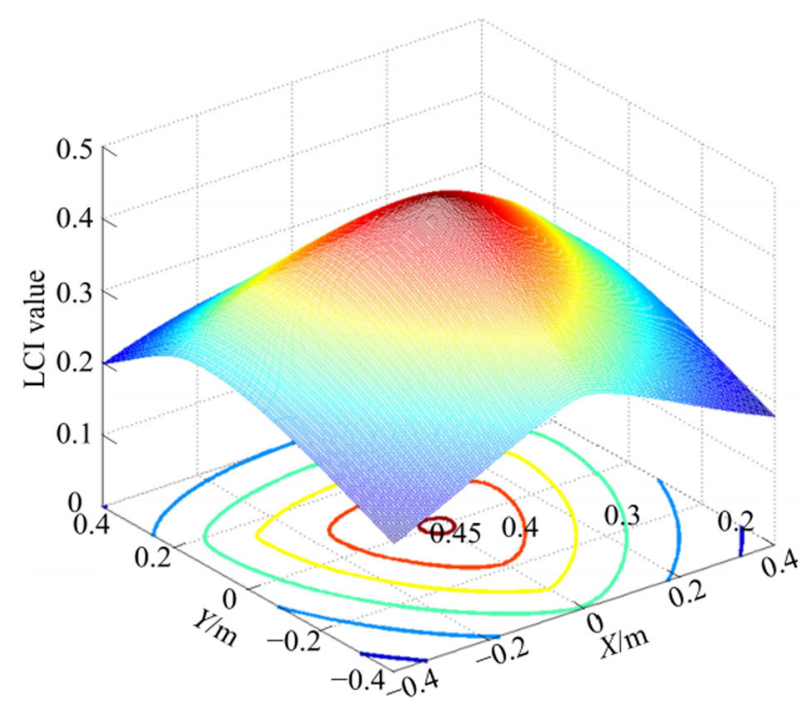

Figure 13 LCI atlas of the Stewart parallel manipulator

radius $r$ is adopted as the characteristic length to deduce the normalized Jacobian matrix.

Generally, overall trends of LCI and OLTI atlases for the Stewart parallel manipulator are similar, as illustrated in Figures 12 and 13. Both LCI and OLTI values decrease while the end effector moves away from the center of the workspace, which indicates that the transmission performance becomes worse. The minimum and maximum values of OLTI are 0.146 and 0.636 respectively. The minimum and maximum values of LCI are 0.178 and 0.456 respectively. The value variation of OLTI (0.49) is greater than that of the LCI $(0.278)$, and the OLTI possesses better performance resolution consequently.

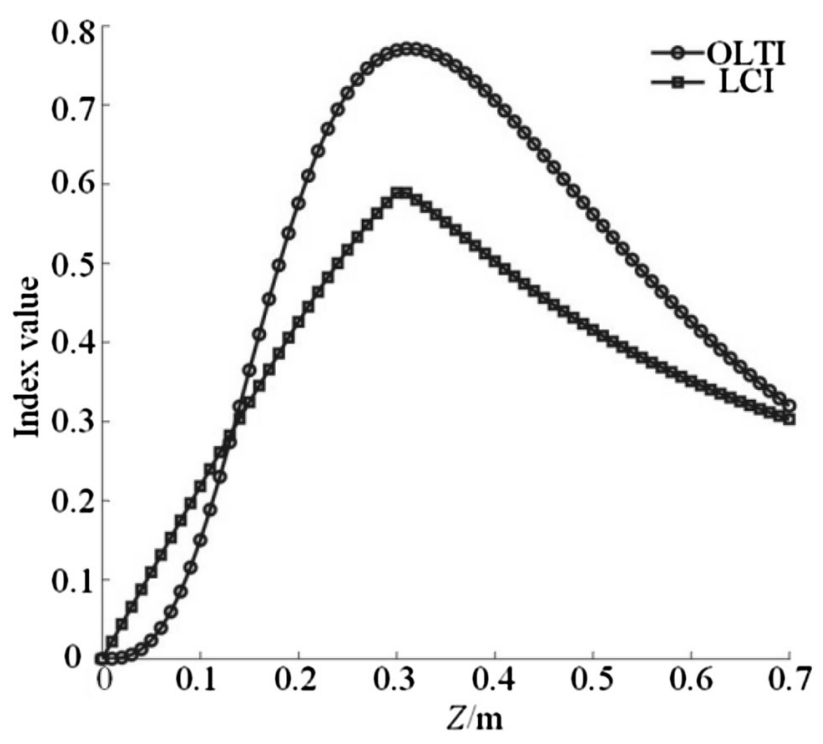

Figure 14 LCI and OLTI value changes along $Z$ axis

Figure 14 illustrates LCI and OLTI value changes when the end effector of the Stewart manipulator moves along $Z$ axis from $0 \mathrm{~m}$ to $0.7 \mathrm{~m}$ with $X$ and $Y$ coordinate values being 0 . The manipulator obtains the best transmission performance when the end effector is located around $Z=0.31 \mathrm{~m}$. The maximum value of OLTI is 0.77 , while the maximum value of LCI is 0.59 . Minimum values of OLTI and LCI are both zero, which indicate the singularity. In the left region, with the end effector approaching the base, both index values decrease, and the value declining speed is faster than that in the right region when the end effector moves away from the base.

Figure 15 indicates LCI and OTLI value changes when the end effector rotates along $C$ axis with angle $\gamma$ from $0^{\circ}$ to $90^{\circ}$ and is located at the position of $(0,0,0.45) \mathrm{m}$. Both LCI and OTLI values gradually decrease. When the rotation angle $\gamma$ equals $90^{\circ}$, values of OTLI and LCI are both 0 , which means the Stewart parallel manipulator is at its singular pose.

\section{Conclusions}

(1) The matrix orthogonal degree is adopted to evaluate the transmission performance of the parallel manipulators. Transmission indices of branch, end effector, and configuration for the fully parallel manipulator are proposed, and a new index system is established.

(2) The BTI is proposed to evaluate the transmissibility of each limb, and transmission performance analysis on typical branches mostly adopted in parallel 


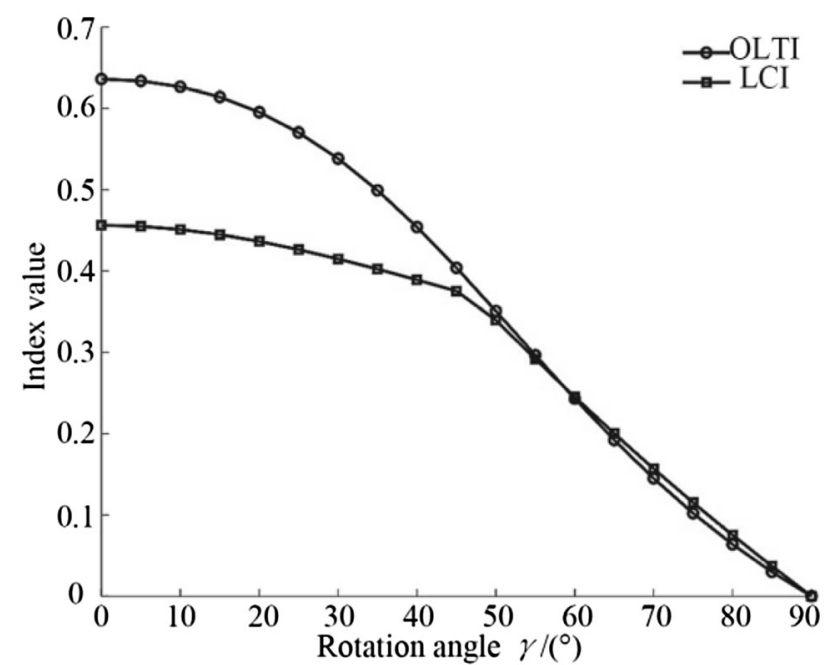

Figure 15 LCI and OLTI value changes along $C$ axis

manipulators are carried out. Specifically, for the branch composed of actuated pendulum and passive two-force bar, such as $\underline{\text { RUS and }}$ RRR, $\Gamma_{\mathrm{BTI} j}=\Gamma_{\mathrm{JTI} j 2}=\sqrt{1-\operatorname{ort}\left(\boldsymbol{Y}_{j 2}\right)^{2}}=\operatorname{ort}\left(\boldsymbol{L}_{j}\right)$. If the branch is composed of actuated prismatic joint and passive two-force bar, such as PUS and PRS, $\Gamma_{\mathrm{BTI} j}=\Gamma_{\mathrm{JTI} 2}=\sqrt{1-\operatorname{ort}\left(\boldsymbol{Y}_{j 2}\right)^{2}}=\sqrt{1-\operatorname{ort}\left(\boldsymbol{L}_{j}\right)^{2}}$. When the actuated two-force branch is adopted, such as UPS and RPS, the BTI value is the constant 1.

(3) The OLTI is defined to evaluate the transmissibility of the fully parallel manipulator. Transmission performances of 5R and Stewart parallel manipulators are analyzed to illustrate the analysis method and the proposed frame-free indices. Compared with the traditional LCI, proposed OLTI possesses better resolution for transmission performance, without intersection phenomenon in the performance contour atlas.

(4) Proposed indices are relative to the singularity. When the JTI value is zero, the BTI value of the joint located branch is zero, and the reverse singularity occurs. On the other side, when the ETI value is zero, the forward singularity appears. Thus, if either BTI or ETI values is zero, the parallel mechanism is at its singular pose. If both BTI and ETI values are zero, the duplex singularity happens.

(5) Simulation and analysis results show that the proposed indices possess better performance resolution than the LCI. Besides, they are frame-free with unified finite range from 0 to 1 , and dimensionally homogeneous with clear physical meaning.
Open Access This article is distributed under the terms of the Creative Commons Attribution 4.0 International License (http://crea tivecommons.org/licenses/by/4.0/), which permits unrestricted use, distribution, and reproduction in any medium, provided you give appropriate credit to the original author(s) and the source, provide a link to the Creative Commons license, and indicate if changes were made.

\section{References}

1. Z F Shao, X Q Tang, L P Wang. Dynamic verification experiment of the Stewart parallel manipulator. International Journal of Advanced Robotic Systems, 2015, 12(10): 144-153.

2. Z F Shao, X Q Tang, X Chen, et al. Inertia match research of reconfigurable planar 3-RRR parallel manipulator. Chinese Journal of Mechanical Engineering, 2009, 22(6): 791-799.

3. Y B Ni, B Zhang, Y P Sun, et al. Accuracy analysis and design of A3 parallel spindle head. Chinese Journal of Mechanical Engineering, 2016, 29(2): 239-249.

4. K J Murthy, V Waldron. Position kinematics of the generalized lobster arm and its series-parallel dual. Journal of Mechanical Design, 1992, 114(3): 406-413.

5. X Q Tang, J S Wang, M Gao. Kinematic calibration of gantry hybrid machine tool based on estimation error and local measurement information. International Journal of Advanced Manufacturing Technology, 2005, 26(4): 382-90.

6. Z F Shao, X Q Tang, L P Wang, et al. A fuzzy PID approach or the vibration control of the FSPM. International Journal of Advanced Robotic Systems, 2013, 10(59): 1-8.

7. $\mathrm{D} \mathrm{S} \mathrm{Wu}, \mathrm{H} \mathrm{B} \mathrm{Gu}$. Adaptive sliding control of six-DOF flight simulator motion platform. Chinese Journal of Aeronautics, 2007, 20(5): 425-433.

8. J Mo, Z F Shao, L W Guan, et al. Dynamic performance analysis of the X4 high-speed pick-and-place parallel robot. Robotics and Computer-Integrated Manufacturing, 2017, 46: 48-57.

9. F Pierot, C Reynaud, A Fournier. DELTA: a simple and efficient parallel robot. Robotica, 1990, 8(2): 105-109.

10. J P Merlet. Parallel robots. Netherlands: Springer Science \& Business Media, 2012.

11. T Huang, M X Wang, S F Yang, et al. Force/motion transmissibility analysis of six degree of freedom parallel mechanisms. Journal of Mechanisms and Robotics, 2014, 6(3): 010.1-0101.5

12. J Angeles. Fundamentals of robotic mechanical systems. New York: Springer-Verlag, 2002.

13. Z F Shao, X Q Tang, L P Wang, et al. Atlas based kinematic optimum design of the Stewart parallel manipulator. Chinese Journal of Mechanical Engineering, 2015, 28(1): 20-28.

14. Z F Shao, X Q Tang, L P Wang. Optimum design of 3-3 Stewart platform considering inertia property. Advance in Mechanical Engineering, 2013, 249121: 1-10.

15. C Gosseline, J Angeles. A global performance index for the kinematic optimization of robotic manipulators. Journal of Mechanical Design, 1991, 113(3): 220-226.

16. J K Salisbury, J J Craig. Articulated hands force control and kinematic issues. The International journal of Robotics Research, 1982, 1(1): 4-17.

17. J P Merlet. Jacobian, manipulability, condition number, and accuracy of parallel robots. Journal of Mechanical Design, 2006, 128(1): 199-206.

18. J Angeles. Is there a characteristic length of a rigid-body displacement. Mechanism and Machine Theory, 2006, 41(8): 884-896. 
19. W A Khan, J Angeles. The kinematic optimization of robotic manipulators: the inverse and direct problems. ASME Journal of Mechanical Design, 2006, 128(1): 168-178.

20. H T Liu, T Huang, D G. Chetwynd. A method to formulate a dimensionally homogeneous Jacobian of parallel manipulator. IEEE Transaction on Robotic, 2011, 27(1): 150-156.

21. O Ma, J Angeles. Optimum architecture design of platform manipulators. International Conference on Advanced Robotics, Pisa, Italy, July 29-31, 1991: 1130-1135.

22. X J Liu, C Wu, J Wang. A new approach for singularity analysis and closeness measurement to singularities of parallel manipulators. Journal of Mechanisms and Robotics, 2012, 4(4): 041001.1- 041001.10.

23. P Voglewede, U I Ebert. Measuring "closeness" to singularities for parallel manipulators. IEEE International Conference on Robotics and Automation, Barcelona, Spain, April 18-22, 2005: 4539-4544.

24. J Wang, C Wu, X J Liu. Performance evaluation of parallel manipulators: Motion/force transmissibility and its index. Mechanism and Machine Theory, 2010, 45(10): 1462-1476.

25. L Zheng, J Angeles. The properties of constant branch for-bar linkages and their applications. ASME transaction on Mechanical Design, 1992, 114: 574-579.

26. S S Balli, S Chand. Transmission angle in mechanism (Triangle in mesh). Mechanism and Machine Theory, 2002, 37(2): 175-195.

27. T Huang, $\mathrm{Z} \mathrm{Li}, \mathrm{M} \mathrm{Li}$, et al. Conceptual design and dimensional synthesis of a novel 2-DOF translational parallel robot for pickand-place operations. Journal of Mechanical Design, 2004, 126(3): 449-455.

28. X J Liu, C Wu, J S Wang. A new index for the performance evaluation of parallel manipulators: a study on planar parallel manipulators. World Congress on Intelligent Control and Automation, Chongqing, China, June 25-27, 2008: 353-357.

29. R E Philipp, F Freudenstein. Synthesis of two-degree-of-freedom linkages - a feasibility study of numerical methods of synthesis of bivariate function generators. Journal of Mechanisms, 1966, 1(1): 9-21.

30. L M Zhang, J P Mei, X M Zhao, et al. Dimensional synthesis of the Delta robot using transmission angle constrains. Robotica, 2012, 30: 343-349.

31. M Zoppi, D Zlatanov, R Molfino. On the velocity analysis of interconnected chains mechanisms. Mechanism and Machine Theory, 2006, 41(11): 1346-1358.

32. J Wu, T M Li, X J Liu, et al. Optimal kinematic design of a 2-DOF planar parallel manipulator. Tsinghua Science \& Technology, 2007, 12(3): 269-275.
33. R S Ball. A Treatise on the Theory of Screws. Cambridge MA: Cambridge University Press, 1998.

34. F Freudenstein, L S Woo. Kinematic analysis of spatial mechanisms by means of screw coordinates. Part 2-Analysis of spatial mechanisms. Journal of Manufacturing Science and Engineering, 1971, 93(1): 67-73.

35. G Sutherland, B Roth. A transmission index for spatial mechanisms. Journal of Manufacturing Science and Engineering, 1973, 95(2): 589-597.

36. M J Tsai, H W Lee. Generalized evaluation for the transmission performance of mechanisms. Mechanism and Machine Theory, 1994, 29(4): 607-618.

37. C Chen, J Angeles. Generalized transmission index and transmission quality for spatial linkages. Mechanism and Machine Theory, 2007, 42(9): 1225-1237.

38. X Chen, X J Liu, F G Xie, et al. A comparison study on motion/force transmissibility of two typical 3-DOF parallel manipulators: Sprint Z3 and A3 tool heads. International Journal of Advanced Robotic System, 2014, 11(5): 1-10.

39. A Ben-Israel. An application of the matrix volume in probability. Linear Algebra and Its Applications, 2000, 321(1): 9-25.

Zhu-Feng Shao, born in 1983, is currently an associate professor at Department of Mechanical Engineering, Tsinghua University, China. $\mathrm{He}$ received his $\mathrm{PhD}$ degree from Tsinghua University, China, in 2011. His research interests include design, dynamic analysis and control of the parallel manipulator and the cable robot. Tel: +86-1062794598; E-mail: shaozf@mail.tsinghua.edu.cn

Jiao Mo, born in 1992, is currently a master candidate at Department of Mechanical Engineering, Tsinghua University, China. He received his bachelor degree from Tsinghua University, China, in 2015. His research interests include high-speed parallel robot and machine tool. E-mail: mjmojiao@126.com

Xiao-Qiang Tang, born in 1973, is currently a professor and a $\mathrm{PhD}$ candidate supervisor at Department of Mechanical Engineering, Tsinghua University, China. His main research interests include parallel manipulator, cable robots, and motion control. E-mail: tangxq@mail.tsinghua.edu.cn

Li-Ping Wang, born in 1967, is currently a professor and a $\mathrm{PhD}$ candidate supervisor at Department of Mechanical Engineering, Tsinghua University, China. His main research interests lie in the advanced manufacturing equipment. E-mail: lpwang@mail.tsinghua.edu.cn 